\title{
Value-based imaging: Combining coronary artery calcium with myocardial perfusion imaging
}

\author{
Daniel S. Berman, MD, FACC, ${ }^{a}$ and Alan Rozanski, MD, FACC ${ }^{b, c}$ \\ a Department of Imaging, Cedars-Sinai Medical Center, Cedars-Sinai Heart Institute, Los Angeles, \\ CA \\ b Division of Cardiology, Mt Sinai St. Luke's and Roosevelt Hospital, Mount Sinai Heart, New \\ York, NY \\ ${ }^{c}$ Icahn School of Medicine at Mount Sinai, New York, NY
}

Received Jul 21, 2016; accepted Jul 22, 2016

doi: 10.1007/s12350-016-0630-0

The unsustainable rising cost of healthcare has thrust us into an era in which the use of medical tests must be of proven clinical value. For diagnostic cardiac tests, this means determining if testing leads to some combination of improving outcomes or cost savingsthe new era of "value-based imaging." Notably, in this regard, it is not the result of a test which changes outcomes, but the management that occurs based on that result.

Radionuclide stress myocardial perfusion imaging (MPI) has been a routine part of the cardiology landscape for approximately 40 years. Its use during this time emerged from its proven ability to predict patient outcomes. How might this test fair best in the "new marketplace" in which the use of tests will now depend on their ability to alter patient outcomes?

The answer to this question is being affected by three important trends. First, since the late 1960s, the clinical presentation of coronary artery disease has markedly changed. While still the nation's leading cause of death, the annualized mortality rate of heart disease has decreased by $70 \%$, myocardial infarction is both less frequent and less severe, and even the frequency of angina is diminishing. From 1991 to 2009, we noted a concomitant marked decline in the frequency of inducible myocardial ischemia on SPECT-MPI among diagnostic patients in the Cedars-Sinai experience, falling from a frequency of $30 \%$ in 1991 to only $5 \%$ in 2009. ${ }^{1}$ Recent experiences in other large datasets, such

\footnotetext{
Reprint requests: Daniel S. Berman, MD, FACC, Department of Imaging, Cedars-Sinai Medical Center, Cedars-Sinai Heart Institute, Los Angeles, CA; bermand@cshs.org

J Nucl Cardiol 2016;23:939-41.

$1071-3581 / \$ 34.00$

Copyright (C) 2016 American Society of Nuclear Cardiology.
}

as the patients recruited into the WOMEN ${ }^{2}$ and PRO$\mathrm{MISE}^{3}$ trials have also demonstrated a concurrent low frequency of inducible myocardial ischemia as well as of obstructive CAD. Further, recent reports have also been documented that the likelihood of obstructive CAD is now far lower than that predicted by the standard approaches which are based on data from the 1970's., Thus, a large majority of SPECT-MPI studies within diagnostic populations can now be expected to be normal. While the finding of a normal SPECT-MPI study without anatomic imaging may reduce referral to invasive coronary angiography (ICA), for the most part, it has little effect on patient management. ${ }^{5}$

Second, studies involving concomitant SPECT-MPI and coronary artery calcium (CAC) scanning have revealed that among patients referred for radionuclide stress testing, the frequency of abnormal CAC studies among patients with normal nuclear studies is quite high. In our earliest experience in this regard, among 1,119 patients with normal SPECT-MPI studies, 78\% had evidence of CAC and in $>30 \%$ of patients, the CAC score was $>400$, indicative of extensive atherosclerosis. ${ }^{6}$ A limitation of radionuclide MPI-stress tests in general-is their inability to detect subclinical atherosclerosis, which is common among patients who are referred for cardiac stress testing. If the report were to include the presence and amount of CAC, i.e., adding anatomic imaging, greater change in management may follow. ${ }^{7}$ Further, there is increasing awareness that SPECT-MPI frequently underestimates the extent of obstructive CAD and may misclassify patients with angiographic highrisk disease. ${ }^{8}$ Knowledge of the presence of extensive coronary calcification could improve these assessments by better establishing the pre-scan likelihood of CAD as well as of high-risk CAD. 
Third, with respect to reporting risk, when the focus with radionuclide stress testing was previously simply aimed at short-term risk assessment, generally involving 2 to 3 years of follow-up, the test was famously known for producing a "warranty period" of low risk. We have subsequently learned, however, that there is heterogeneity in long-term outcomes among patients with normal SPECT-MPI studies, with risk increasing with both rising number of CAD risk factors ${ }^{9-11}$ and with increasing CAC scores. ${ }^{12,13}$ Accordingly, we have recently suggested that overall risk assessment, which can help provide for better disease management, can be complemented by identifying both short-term and longterm risk and conveying this information on nuclear stress test reports. ${ }^{14}$

Given these trends, use of CAC scanning before or combining it with SPECT- or PET-MPI can lead to a change in the clinical management to a greater degree than the use of SPECT-MPI in isolation in the following ways:

(1) Improved selection of patients for stress testing In the current era of low ischemic frequency and overestimation of the likelihood of obstructive CAD, methods to more reliably identify which patients truly need cardiac stress tests is desirable. Low CAC scores diminish the likelihood of obstructive CAD and inducible myocardial ischemia, ${ }^{6,11,15}$ even among patients with an intermediate likelihood of CAD. ${ }^{16}$ Thus, CAC scanning can reduce the number of patients who require SPECT-MPI due to low CAC scores. Conversely, the finding of a very extensive CAC score may lead to appropriate testing of patients who may not otherwise been identified as being at high risk. As a note of caution, the CAC score "threshold" for ischemia may be lower among patients with typical angina, ${ }^{16}$ and more study is required regarding this important subgroup of patients. More study is also needed to assess how other clinical variables shape the relationship between CAC scores and inducible myocardial ischemia. $^{17}$

(2) Improved diagnosis In 2016, the goal of diagnosis in patients with chest pain syndromes should not merely be the presence of obstructive CAD, but rather the identification of any CAD (i.e., any coronary atherosclerosis). CAC scanning provides identification of the presence and extent of subclinical atherosclerosis in a large percentage of patients who now have normal MPI studies during diagnostic work-up. Knowledge of the CAC in conjunction with a SPECT-MPI study could provide similar improvements in diagnosis, resulting in similar guidance of the intensity of medical therapy to that provided by coronary CTA. Moreover, CAC scanning can also be combined with other clinical methods to improve the Bayesian estimation of obstructive CAD. ${ }^{18}$

(3) Improved risk assessment CAC scanning can improve overall risk assessment in two ways. First, in patients with a normal MPI scan, short-term risk has been shown to be low regardless of the CAC score, ${ }^{19}$ but after 5 to 6 years of follow-up, risk becomes heterogeneous, varying according to the magnitude of CAC abnormality. ${ }^{12}$ Second, assessment of CAC score improves overall risk assessment across the spectrum of MPI abnormality. ${ }^{12,13}$ Improved risk assessment could likely lead to wiser decisions as to the need for aggressive treatment vs minimization of wasteful spending. Of note in this regard, in the EISNER trial, randomization to risk factor counseling with CAC scanning (vs risk factor counseling without CAC scanning) led to improved patient risk factor profiles without increasing downstream testing or overall incurred medical costs during four years of follow-up. ${ }^{4}$

(4) Improved test interpretation Knowledge of the CAC score in individual patients frequently provides great assistance in the decision as to whether to report the otherwise equivocal MPI scan as normal or abnormal-and often increases interpreter certainty. ${ }^{20}$ Improved interpretation should decrease the proportion of patients referred for ICA following SPECT who are found to have nonobstructive CAD and improve the identification of patients with high-risk CAD. These factors could improve the use of SPECT-MPI as a gatekeeper to ICA.

(5) Improved physician/patient management The CAC scan can affect the degree to which the patient implements heart-healthy behaviors following testing. $^{21,22}$

PET-MPI is becoming increasingly common as the modality for radionuclide MPI, and the vast majority of PET-MPI studies are performed with PET/CT systems with which CAC scanning at the time of PET-MPI is readily available. SPECT-CT systems with similar capabilities for CAC assessments to those of the PET/ CT systems are becoming increasingly used. Further, with these systems, the extent of CAC can be estimated with reasonable accuracy from the attenuation scans routinely performed for the PET or SPECT study. ${ }^{23}$ And with the marked recent increase in the use of CAC scanning for the guiding aggressiveness of medical therapy, CAC scans that are separate from the MPI study are now commonly available.

So in which setting should routine use of CAC scanning with MPI be considered? Given the five advantages noted above, perhaps in all patients without 
known CAD who are referred for these procedures. The combination of functional and anatomic assessments could increase the frequency of beneficial changes in patient management following testing, potentially allowing MPI to compete more effectively with coronary CTA in patients with an intermediate likelihood of CAD with respect to value-based imaging. The combined assessments should become the standard in future clinical trials involving nuclear MPI examinations.

\section{Acknowledgment}

Supported in part by a grant from the Adelson Family Foundation and the Diane and Guilford Glazer Foundation.

\section{Disclosures}

None.

\section{References}

1. Rozanski A, Gransar H, Hayes SW, et al. Temporal trends in the frequency of inducible myocardial ischemia during cardiac stress testing: 1991 to 2009. J Am Coll Cardiol 2013;61:1054-65.

2. Shaw LJ, Mieres JH, Hendel RH, et al. Comparative effectiveness of exercise electrocardiography with or without myocardial perfusion single photon emission computed tomography in women with suspected coronary artery disease: results from the What Is the Optimal Method for Ischemia Evaluation in Women (WOMEN) trial. Circulation 2011;124:1239-49.

3. Douglas PS, Hoffmann U, Patel MR, et al. Outcomes of anatomical versus functional testing for coronary artery disease. $\mathrm{N}$ Engl J Med 2015;372:1291-300.

4. Cheng VY, Berman DS, Rozanski A, et al. Performance of the traditional age, sex, and angina typicality-based approach for estimating pretest probability of angiographically significant coronary artery disease in patients undergoing coronary computed tomographic angiography: results from the multinational coronary CT angiography evaluation for clinical outcomes: an international multicenter registry (CONFIRM). Circulation 2011;124(242332): $1-8$.

5. Hachamovitch R, Nutter B, Hlatky MA, et al. Patient management after noninvasive cardiac imaging results from SPARC (Study of myocardial perfusion and coronary anatomy imaging roles in coronary artery disease). J Am Coll Cardiol 2012;59:462-74.

6. Berman DS, Wong ND, Gransar H, et al. Relationship between stressinduced myocardial ischemia and atherosclerosis measured by coronary calcium tomography. J Am Coll Cardiol 2004;44:923-30.

7. Bybee KA, Lee J, Markiewicz R, et al. Diagnostic and clinical benefit of combined coronary calcium and perfusion assessment in patients undergoing PET/CT myocardial perfusion stress imaging. J Nucl Cardiol 2010;17:188-96.

8. Berman DS, Kang X, Slomka PJ, et al. Underestimation of extent of ischemia by gated SPECT myocardial perfusion imaging in patients with left main coronary artery disease. J Nucl Cardiol 2007;14:521-8.

9. Supariwala A, Uretsky S, Depuey EG, et al. Influence of mode of stress and coronary risk factor burden upon long-term mortality following normal stress myocardial perfusion single-photon emission computed tomographic imaging. Am J Cardiol 2013;111:846-50.

10. Supariwala A, Uretsky S, Singh P, et al. Synergistic effect of coronary artery disease risk factors on long-term survival in patients with normal exercise SPECT studies. J Nucl Cardiol 2011;18:207-14 quiz 217.

11. Rozanski A, Gransar H, Min JK, et al. Long-term mortality following normal exercise myocardial perfusion SPECT according to coronary disease risk factors. J Nucl Cardiol 2014;21:341-50.

12. Chang SM, Nabi F, Xu J, et al. The coronary artery calcium score and stress myocardial perfusion imaging provide independent and complementary prediction of cardiac risk. J Am Coll Cardiol 2009;54:1872-82.

13. Engbers EM, Timmer JR, Ottervanger JP, Mouden M, Knollema $\mathrm{S}$, Jager PL. Prognostic value of coronary artery calcium scoring in addition to single-photon emission computed tomographic myocardial perfusion imaging in symptomatic patients. Circulation 2016;9(5):e003966.

14. Rozanski A, Berman DS. Long-term risk assessment after the performance of stress myocardial perfusion imaging. Cardiol Clin. 2016;34:87-99.

15. Rozanski A, Uretsky S, Berman DS. Use of coronary artery calcium scanning as a triage for cardiac ischemia testing. J Nucl Cardiol 2016. doi:10.1007/s12350-016-0405-7.

16. Rozanski A, Gransar H, Wong ND, et al. Use of coronary calcium scanning for predicting inducible myocardial ischemia: Influence of patients' clinical presentation. J Nucl Cardiol 2007;14:669-79.

17. Bavishi C, Argulian E, Chatterjee S, Rozanski A. CACS and the frequency of stress-induced myocardial ischemia during MPI: a meta-analysis. JACC Cardiovasc Imaging 2016;9(5):580-9.

18. Budoff MJ, Diamond GA, Raggi P, et al. Continuous probabilistic prediction of angiographically significant coronary artery disease using electron beam tomography. Circulation 2002;105:1791-6.

19. Rozanski A, Gransar H, Wong ND, et al. Clinical outcomes after both coronary calcium scanning and exercise myocardial perfusion scintigraphy. J Am Coll Cardiol 2007;49:1352-61.

20. Uretsky S, Cohen R, Argulian E, et al. Combining stress-only myocardial perfusion imaging with coronary calcium scanning as a new paradigm for initial patient work-up: an exploratory analysis. J Nucl Cardiol 2015;22:89-97.

21. Mamudu HM, Paul TK, Veeranki SP, Budoff M. The effects of coronary artery calcium screening on behavioral modification, risk perception, and medication adherence among asymptomatic adults: a systematic review. Atherosclerosis 2014;236:338-50.

22. Rozanski A, Gransar H, Shaw LJ, et al. Impact of coronary artery calcium scanning on coronary risk factors and downstream testing the EISNER (Early Identification of Subclinical Atherosclerosis by Noninvasive Imaging Research) prospective randomized trial. J Am Coll Cardiol 2011;57:1622-32.

23. Einstein AJ, Johnson LL, Bokhari S, et al. Agreement of visual estimation of coronary artery calcium from low-dose CT attenuation correction scans in hybrid PET/CT and SPECT/CT with standard Agatston score. J Am Coll Cardiol 2010;56:1914-21. 